\title{
Analysis of Gut Microbiota in Rheumatoid Arthritis Patients: Disease-Related Dysbiosis and Modifications Induced by Etanercept
}

\author{
Andrea Picchianti-Diamanti ${ }^{1, *,+} \mathbb{1}$, Concetta Panebianco ${ }^{2, \dagger}{ }^{+}$Simonetta Salemi ${ }^{1}$, \\ Maria Laura Sorgi ${ }^{1}$, Roberta Di Rosa ${ }^{1}$, Alessandro Tropea ${ }^{1}$, Mayla Sgrulletti ${ }^{1}$, \\ Gerardo Salerno ${ }^{1}$, Fulvia Terracciano ${ }^{2}$, Raffaele $D^{\prime}$ Amelio ${ }^{1}{ }^{1}$, Bruno Laganà ${ }^{1}$ and \\ Valerio Pazienza ${ }^{2, *}$ \\ 1 Department of Clinical and Molecular Medicine, Sant'Andrea University Hospital, Sapienza University of \\ Rome, 00185 Rome, Italy; s.salemi@gmail.com (S.S.); marialaura.sorgi@uniroma1.it (M.L.S.); \\ roberta.dirosa@uniroma1.it (R.D.R.); alessandro.tropea3@gmail.com (A.T.); \\ Mayla.sgrulletti@uniroma1.it (M.S.); Gerardo.salerno@uniroma1.it (G.S.); \\ raffaele.damelio@uniroma1.it (R.D.); bruno.lagana@uniroma1.it (B.L.) \\ 2 Gastroenterology Unit, IRCCS “Casa Sollievo della Sofferenza” Hospital, Viale dei Cappuccini, 1, \\ 71013 San Giovanni Rotondo, Italy; panebianco.c@gmail.com (C.P.); terracciano74@hotmail.com (F.T.) \\ * Correspondence: andrea.picchiantidiamanti@uniroma1.it (A.P.-D.); v.pazienza@operapadrepio.it (V.P.) \\ $\dagger$ These authors contributed equally to the manuscript. \\ $\ddagger$ Retired.
}

Received: 23 August 2018; Accepted: 25 September 2018; Published: 27 September 2018

\begin{abstract}
A certain number of studies were carried out to address the question of how dysbiosis could affect the onset and development of rheumatoid arthritis (RA), but little is known about the reciprocal influence between microbiota composition and immunosuppressive drugs, and how this interaction may have an impact on the clinical outcome. The aim of this study was to characterize the intestinal microbiota in a groups of RA patients treatment-naïve, under methotrexate, and/or etanercept (ETN). Correlations between the gut microbiota composition and validated immunological and clinical parameters of disease activity were also evaluated. In the current study, a $16 \mathrm{~S}$ analysis was employed to explore the gut microbiota of 42 patients affected by RA and 10 healthy controls. Disease activity score on 28 joints (DAS-28), erythrocyte sedimentation rate, C-reactive protein, rheumatoid factor, anti-cyclic citrullinated peptides, and dietary and smoking habits were assessed. The composition of the gut microbiota in RA patients free of therapy is characterized by several abnormalities compared to healthy controls. Gut dysbiosis in RA patients is associated with different serological and clinical parameters; in particular, the phylum of Euryarchaeota was directly correlated to DAS and emerged as an independent risk factor. Patients under treatment with ETN present a partial restoration of a beneficial microbiota. The results of our study confirm that gut dysbiosis is a hallmark of the disease, and shows, for the first time, that the anti-tumor necrosis factor alpha (TNF- $\alpha$ ) ETN is able to modify microbial communities, at least partially restoring a beneficial microbiota.
\end{abstract}

Keywords: microbiota; rheumatoid arthritis; anti-TNF- $\alpha$; methotrexate; etanercept; disease activity

\section{Introduction}

The human intestinal microbiota is a complex microcosm composed of more than 1000 different bacterial species, archaea, fungi, and viruses [1]. There is growing knowledge that these bacteria are not only involved in the digestion and absorption of food, but they can also exert a protective function 
by preventing adherence of pathogenic bacteria to the mucosal layer, and they play a pivotal role in modulating the innate and acquired immunity of the host [2-4].

Recent advances in sequencing technologies led to a deep characterization of the human gut microbiota in healthy subjects. This enabled the investigation of modifications in the structure of gut commensal communities (called dysbiosis), which could be involved in the onset and maintenance of different chronic autoimmune diseases, such as inflammatory bowel diseases (IBD) and arthritis $[5,6]$. Dysbiosis could lead to alterations in the intestinal epithelial cell layer with an increased exposure to a variety of bacteria and bacterial products leading to a chronic antigenic stimulation, spreading of inflammatory mediators, and T cell activation [7,8].

It was recently demonstrated that different environmental factors are involved in the development of both intestinal/oral dysbiosis and arthritis onset and outcome, among which the most relevant are diet, smoking, infections, and drugs [9-12].

Rheumatoid arthritis (RA) is an inflammatory autoimmune disease of unknown etiology, potentially leading to progressive joint destruction and disability. In RA patients, an accumulating body of studies demonstrated a pathogenic role of dysbiosis of the oral microbiota, in particular, an association between Porphyromonas gingivalis, periodontitis, and the generation of citrullinated products was clearly demonstrated [13-15].

On the other hand, data regarding the role of intestinal microbiota in these patients are not conclusive, and no studies address the reciprocal influence between biotechnological immunosuppressants and microbiota on RA outcome.

Etanercept (ETN, a dimeric recombinant fully human fusion protein consisting of a human 75-kDa tumor necrosis factor (TNF) receptor linked to the Fc portion of human immunoglobulin G1 (IgG1)) is one of the five currently available biotechnological agents that target TNF- $\alpha$. ETN proved to be safe and effective in reducing disease activity and limiting the progression of joint damage in RA patients; it can be administered as monotherapy or in combination therapy with methotrexate (MTX) [16].

The aim of this study was to characterize the intestinal microbiota in a group of RA patients treatment-naïve, under MTX, and/or ETN. Correlations between the gut microbiota composition and validated immunological and clinical parameters of disease activity were also evaluated.

\section{Results}

\subsection{Microbiota Profile in Rheumatoid Arthritis (RA) Patients Free of Therapy versus Healthy Controls(HCs)}

Lifestyle factors, as well as demographic, serologic, and clinical parameters, of the four RA treatment groups are shown in Table 1.

No significant differences were observed among the RA groups except for the disease duration, which, as expected, was shorter in the group of naïve and MTX monotherapy patients. HCs were also similar to RA patients regarding lifestyle factors, but they were significantly younger $(p<0.05)$.

After passing quality control filters, a mean of 281,218 sequences per sample were obtained. The assessment of the Shannon index in each sample revealed that $\alpha$-diversity was neither changed in naïve patients compared with the HCs, nor in each treatment group (naïve, ETN, MTX, or ETN plus MTX) relative to patients free of therapy (Figure 1A). Similarly, no significant change in species richness was observed (Figure 1B). 
Table 1. Main demographic, clinical, and serologic data of the 42 rheumatoid arthritis (RA) patients.

\begin{tabular}{|c|c|c|c|c|c|}
\hline Patient Characteristics & Naïve & ETN & MTX & ETN + MTX & $p$-Value \\
\hline Male $n(\%)$ & $1(9)$ & $1(10)$ & $2(18)$ & $2(20)$ & ns \\
\hline Age & 55.7 & 59.8 & 62.3 & 64.6 & ns \\
\hline Varied and balanced diet $n(\%)$ & $10(91)$ & $9(90)$ & $10(91)$ & $9(90)$ & ns \\
\hline Smokers $n(\%)$ & $1(9)$ & $3(30)$ & $1(9)$ & $1(10)$ & ns \\
\hline Disease Duration (years) & 6.4 & 14.8 & 11.2 & 19.9 & $\begin{array}{c}0.007 \text { * } \\
0.07 \\
0.002 \\
0.04\end{array}$ \\
\hline DAS-28 & 4.3 & 3.9 & 4 & 3.7 & ns \\
\hline RF pos $n(\%)$ & $8(73)$ & $6(60)$ & $8(73)$ & $6(60)$ & ns \\
\hline ACPA pos $n(\%)$ & $8(73)$ & $6(60)$ & $8(73)$ & $6(60)$ & ns \\
\hline $\operatorname{ESR}(\mathrm{mm} / \mathrm{h})$ & 27.4 & 28.3 & 22.4 & 22.6 & ns \\
\hline CRP (mg/L) & 5.7 & 6.5 & 5.1 & 6.4 & ns \\
\hline
\end{tabular}

RA = Rheumatoid arthritis; Naïve = patients naïve to immunosuppressants; ETN = etanercept; MTX = methotrexate; $\mathrm{ACPA}=$ anti-citrullinated peptide antibodies; $\mathrm{RF}=$ rheumatoid factor; $\mathrm{ESR}=$ erythrocyte sedimentation rate; $\mathrm{CRP}=$ C-reactive protein; DAS-28 = disease activity score on 28 joints. Data are expressed as means. ${ }^{*}$ Naïve vs. ETN; naïve vs. MTX; naïve vs. ETN + MTX; MTX vs. ETN + MTX. ns = not significant.

A

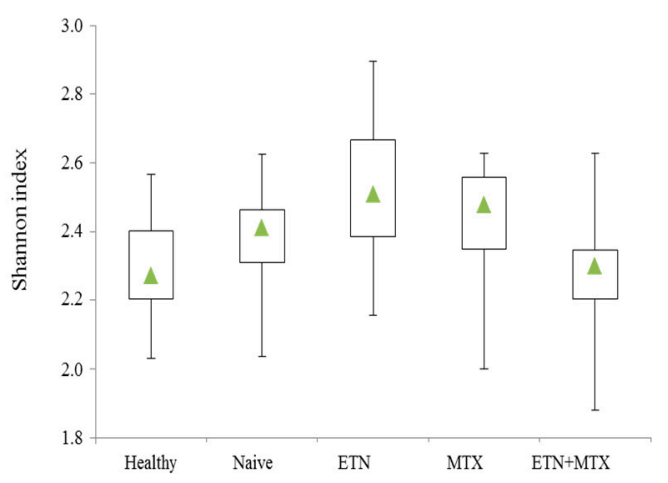

B

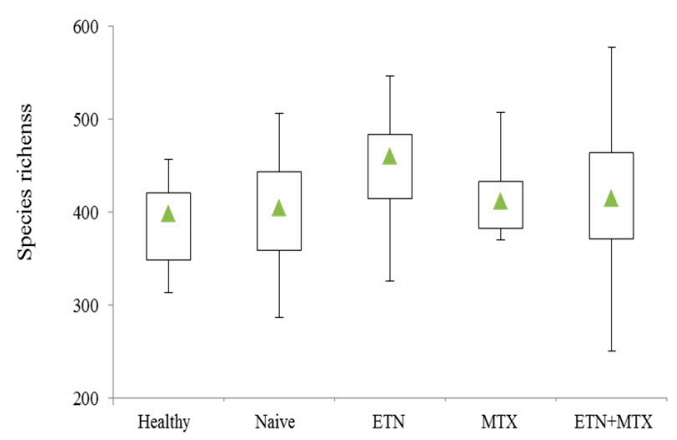

Figure 1. Box plots of Shannon diversity index (A) and species richness (B) of microbiota of healthy controls and different treatment groups of rheumatoid arthritis (RA) patients. The triangle represents the median value.

As a first approach, we compared the gut microbiota composition of the HCs with that of RA patients free of therapy. This analysis revealed that the relative abundance of the microbial phyla was almost unchanged (Figure 2A), while significant differences between the two groups were found at lower taxonomic levels. The most striking alterations were a five-fold increase in the class of Bacilli (Figure 2B) and a seventeen-fold increase in the order of Lactobacillales (Figure 2C) found in RA patients with respect to the controls $(2.89 \pm 3.19$ vs. $0.58 \pm 0.39 ; p=0.035$, and $2.69 \pm 3.15$ vs. $0.15 \pm 0.34 ; p=0.021$, respectively). Significant reductions of the genus Faecalibacterium (12.21 \pm 7.4 vs. $19.72 \pm 4.41 ; p=0.012$ ) (Figure 3B) and its cognate species Faecalibacterium prausnitzii ( $3.23 \pm 3.22$ vs. $7.97 \pm 3.78 ; p=0.006$ ) (Figure 3 C) were also found. Furthermore, significant changes were also observed in the genus Flavobacterium (Figure 3B) and the species Blautia coccoides (Figure 3C), which were both represented in the control group, but were not detected at all in RA naïve patients $(2 \pm 2.44$ vs. $0 \pm 0 ; p=0.013$, and $0.7 \pm 0.76$ vs. $0 \pm 0 ; p=0.006$ ). 

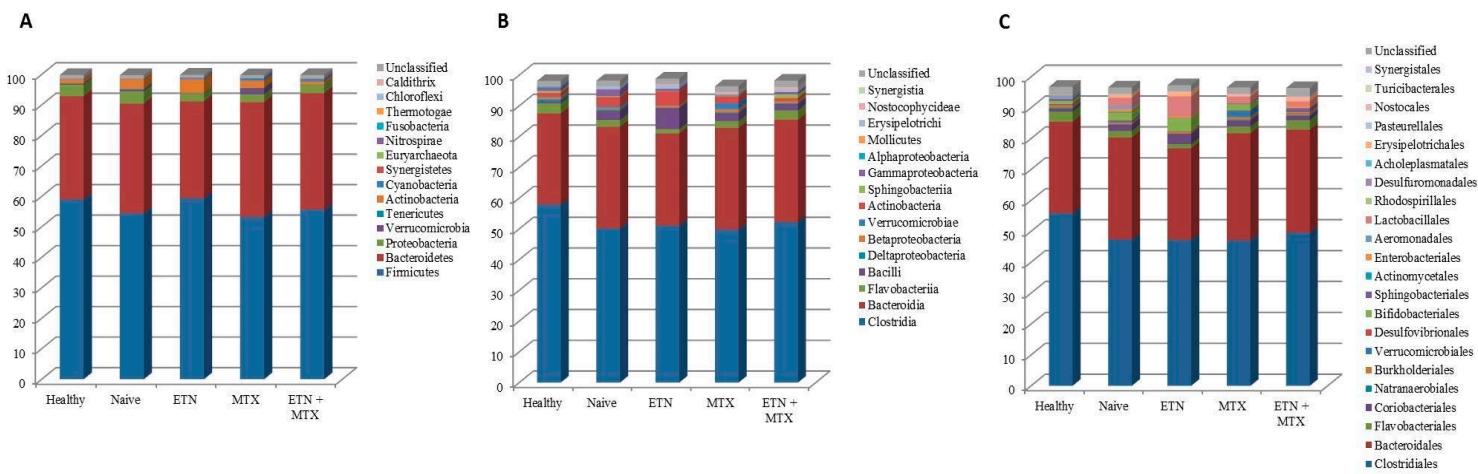

Figure 2. Microbiota composition of healthy controls and different treatment groups of RA patients at the phylum (A), class (B), and order (C) levels. The mean value of the eight top taxonomic classifications at each level is represented.


Figure 3. Microbiota composition of healthy controls and different treatment groups of RA patients at the family (A), genus (B), and species (C) levels. The mean value of the eight top taxonomic classifications at each level is represented.

\subsection{Microbiota Profile in RA Patients Free of Therapy versus Treated Patients}

We next sought to evaluate any difference in microbiota composition of RA patients based on whether they were free of therapy or they received ETN, MTX, or a combination of the two. Mounting evidence supports the existence of a reciprocal connection between drugs and microbiota, which can influence each other and have an impact on therapeutic outcomes [17]. Specifically, MTX was shown to modify microbiota composition, partly restoring the microbial balance altered by the disease [12].

When compared to naïve patients, major changes were observed in the ETN group. The Cyanobacteria significantly increased ( $0.49 \pm 0.5$ vs. $0.08 \pm 0.07 ; p=0.016)$ (Figure $2 \mathrm{~A})$, and the same increase was observed in the Nostocophycideae class (Figure 2B) and the Nostocales order $(0.35 \pm 0.5$ vs. $0 \pm 0 ; p=0.031)$ (Figure $2 C)$, which both belong to the phylum of Cyanobacteria. In detail, Nostocophycideae and Nostocales, which were not represented among the naïve subjects, were instead detected in four out of ten ETN patients. In addition, the class of Deltaproteobacteria $(0.07 \pm 0.23$ vs. $0.57 \pm 0.72 ; p=0.05)$ (Figure $2 \mathrm{~B})$ and the family of Clostridiaceae ( $1.51 \pm 1.76$ vs. $3.97 \pm 3.4 ; p=0.05$ ) (Figure 3A) significantly decreased in the ETN group as compared to the naïve. The only statistically significant alteration found in the MTX group was a decrease in the relative 
abundance of Enterobateriales ( $0.07 \pm 0.24$ vs. $0.85 \pm 1.22 ; p=0.05)$ (Figure $2 C)$, while no significant changes were observed in RA patients upon ETN plus MTX therapy.

\subsection{Association of Microbiota Profile with Clinical Pathological Features in RA Patients}

Finally, we wondered whether microbiota composition could be associated to clinical parameters (i.e., sex, age, disease duration, disease activity Score on 28 joints (DAS-28), rheumatoid factor (RF), anti-cyclic citrullinated peptides antibodies (ACPA), erythrocyte sedimentation rate (ESR) and C-reactive protein (CRP) and lifestyle factors (diet and smoking habits) of the RA patients. For this purpose, correlations were analyzed between microorganisms at each taxonomic level and all the above described parameters (Figure 4).
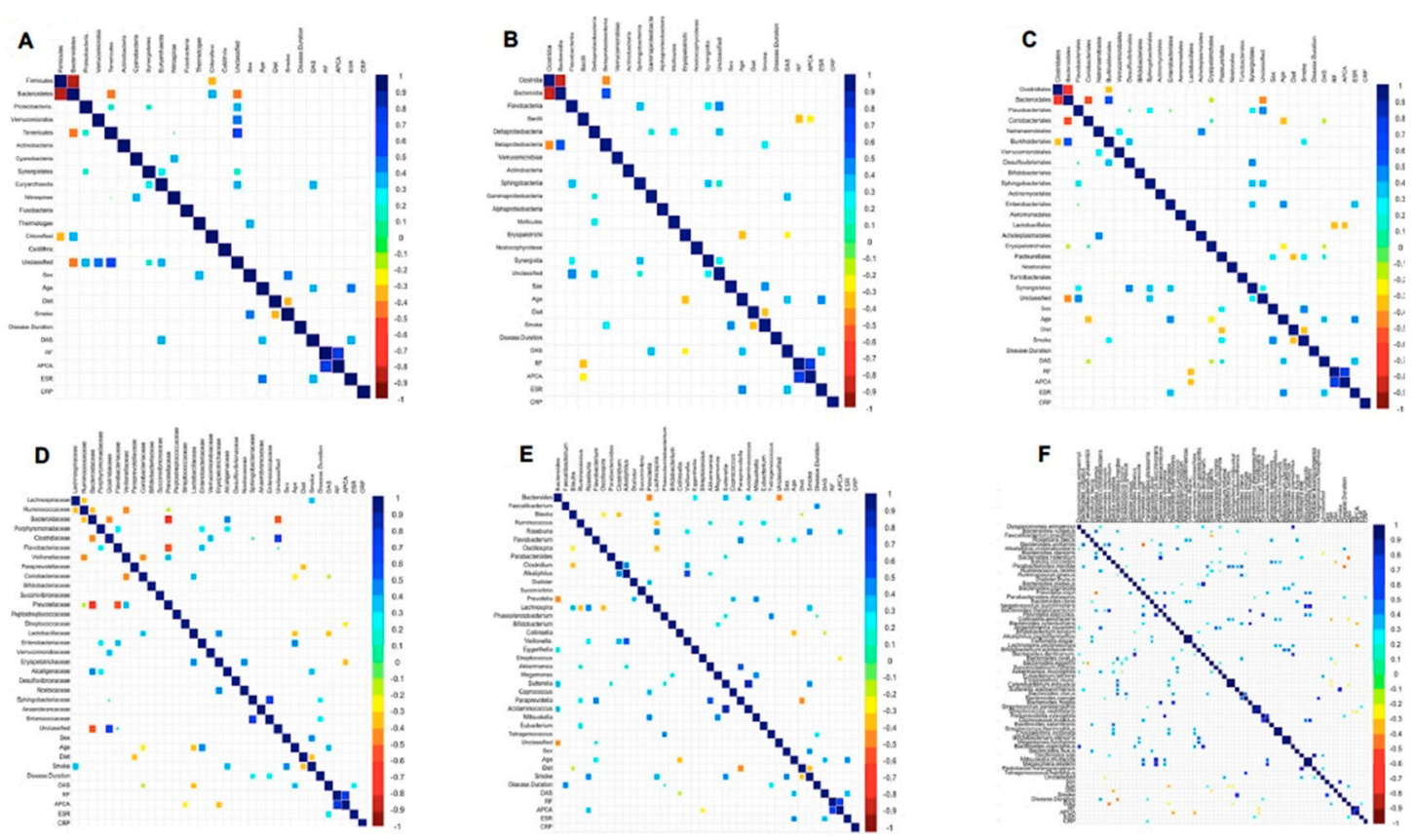

Figure 4. Association of gut microbiota profile with clinical pathological features in RA patients at the phylum (A), class (B), order (C), family (D), genus (E), and species (F) levels.

The Spearman correlation analysis revealed a direct association between the male sex and the abundance of Pasteurellales microbes, while the age of the patients was directly correlated with Enterobacteriales, Enterobacteriaceae, Flavobacterium, Parabacteroides distasonis, and Bacteroides ovatus, and inversely correlated to Erysipelotrichi, Coriobacteriales, Erysipelotrichales, Coriobacteriaceae, Lactobacillaceae, Collinsella, and Collinsella aerofaciens. The disease duration result was positively associated with the species Bacteroides caccae, while it was negatively correlated with Parabacteroides merdae. Interestingly, a direct correlation between DAS and Euryarchaeota, Gammaproteobacteria, Pasteurellales, and Anaerobranca zavarzinii was found, while Erysipelotrichi, Erysipelotrichales, Coriobacteriales, Coriobacteriaceae, Lactobacillaceae, Collinsella, Bacteroides rodentium, and Collinsella aerofaciens were inversely associated with this score. A certain number of correlations were shared by RF and ACPA positivity. In detail, both factors were positively associated with Roseburia and negatively with Bacilli, Lactobacillales, and Streptococcus vestibularis. In addition, ACPA positivity was indirectly correlated with Streptococcaceae, Erysipelotrichaceae, Streptococcus, Bacteroides xylanisolvens, and Lachnospira pectinoschiza. Direct correlations between ESR and Enterobacteriales, Roseburia faecis, and Streptococcus parasanguinis, and between CRP and Parabacteroides distasonis emerged from the analysis. Regarding lifestyle factors, a varied and well-balanced diet was inversely associated with Pasteurellales, Paraprevotellaceae, Paraprevotella, Blautia, Blautia coccoides, and Bacteroides eggerthi, 
while smoking was positively correlated with Betaproteobacteria, Burkholderiales, Pasteurellales, Lachnospiraceae, Alcaligenaceae, Roseburia, Lachnospira, Sutterella, Lachnospira pectinoschiza, Bacteroides denticanum, and Sutterella wadsworthensis.

Additionally, since the variables affecting the microbiota composition in RA are numerous and can be interconnected, a multivariate approach was followed for a more complete understanding of these interactions. This analysis revealed that independent factors affecting the disease were DAS and Euryarchaeota at the phylum level, DAS and Erysipelotrichi at the class level, and only DAS at the order level. On the contrary, no independent factors were found to correlate with the disease at the family, genus, and species levels (Supplementary Material).

\section{Discussion}

In our pilot study, we applied $16 \mathrm{~S}$ analysis to characterize the gut microbiota of RA patients, with a particular interest in the effect of synthetic and biotechnological therapies on its composition. Any significant difference in the Shannon index and in the number of species between healthy and diseased subjects was observed in our study population. No change was observed in richness and diversity when patients were classified on the basis of the treatment they received.

A comparison of the gut microbial populations between healthy and affected individuals pointed out the role of Bacilli in the pathogenesis of RA. Our data are in line with previous studies, in which the related taxa of Lactobacillaceae and Lactobacillus were significantly more abundant in RA animal models and patients than in controls. A study by Liu et al. [18] demonstrated that the quantity and variety of lactobacilli in RA patients was higher than in HCs. A few years later, the same authors described an increase of the Lactobacillaceae family and the Lactobacillus genus in mice susceptible to developing collagen-induced arthritis, with respect to mice resistance, thus suggesting these bacteria as a predisposing factor of the disease [19]. Moreover, Zhang et al. observed an over-representation of Lactobacillus salivarius in the gut and mouth of RA patients, with the highest levels found in people most severely affected [12]. Bacilli (especially Lactobacillus) are generally regarded as friendly bacteria for the host; as such, they are among the most commonly used probiotics. Indeed, the administration of L. casei and L. delbrueckii was shown to alleviate RA symptoms in experimental models [19-22]; on the other hand, L. rhamnosus GG and L. reuteri administration failed to ameliorate the disease in patients [23-25] suggesting that different Lactobacillus species may act differently on RA.

The decreased abundance of Faecalibacterium found in our analysis is in agreement with previous studies carried out on RA [26,27] and other inflammatory conditions [28-30]. The bacteria belonging to this genus are well-known butyrate producers, help in maintaining the integrity and health of the gut epithelial barrier, and exhibit anti-inflammatory properties [28-30]; thus, their decrease may contribute to the onset of an inflammatory status. No previous association of Blautia coccoides (which was depleted in our naïve group) with RA was reported; nonetheless, it was shown that enriching the gut microbiota of systemic lupus erythematosus patients with Blautia coccoides, together with Ruminococcus obeum and Bifidobacterium bifidum, improved the inflammatory status by inducing the production of the immunosuppressive regulatory T cells (Tregs) [31].

As reported by the latest EULAR recommendations, MTX should be considered the first immunosuppressive treatment strategy in patients with RA; in the case of an inadequate response, TNF- $\alpha$ inhibitors or other biotechnological therapies should be started [16]. Zhang et al. previously reported that MTX can affect microbiota composition, partly reversing disease-related dysbiosis [12]. As of now, data on the effect of microbiota in the outcome of patients receiving immunosuppressive biotechnological therapies are limited and only refer to IBD patients. For this reason, we next characterized the gut microbiota of RA patients on different immunosuppressant treatment strategies (ETN, MTX, or ETN plus MTX) and compared it with that of treatment-naive patients.

Interestingly, significant changes were found in patients receiving ETN. The phylum of Cyanobacteria and its cognate Nostocophycideae and Nostocales were enriched in the ETN group. Little is known about the role of these microorganisms in health and disease; nevertheless, Cyanobacteria produce 
secondary metabolites with multiple bioactivities, including anti-inflammatory and immunosuppressant activities [32] which may benefit RA patients. Also, the drop in Deltaproteobacteria caused by ETN could be potentially beneficial if we consider that these microorganisms were found enriched in patients suffering from ulcerative colitis [33] and that Proteobacteria in general are abundant in both intestinal and extra-intestinal inflammatory diseases [34]. Moreover, we observed a decrease in Clostridiaceae upon ETN treatment which could be potentially beneficial since these bacteria were previously found enriched in patients with RA and IBD-associated arthropathy [35]. In patients treated with MTX, our analysis revealed a significant decrease in Enterobacteriales, whose lipopolysaccharides may contribute to inflammation [36], and which were associated with increased intestinal permeability [37], a condition that can be found in RA patients [38].

The results discussed so far further support the evidence of a link between gut microbiota and RA, and show, for the first time, that anti-TNF- $\alpha$ therapy can have a beneficial impact on the microbiota composition.

With the aim of establishing any association between microbial taxa and clinical parameters of the disease, correlation analyses were performed grouping all forty-two RA patients. Among the most remarkable results, the pro-inflammatory Gammaproteobacteria and its cognate order of Pasteurellales displayed a direct correlation with disease activity (DAS-28), while the order of Enterobacteriales showed a positive association with ESR. Interestingly, the phylum of Euryarchaeota was directly correlated with DAS and emerged as an independent risk factor in RA when a multivariate analysis was performed. The function of these microorganisms in humans is still poorly explored, but their increase was observed in another autoimmune disorder, i.e., multiple sclerosis [39].

A positive association between B. caccae and disease duration was also found. A membrane protein of B. caccae, namely outer membrane protein $W(\mathrm{OmpW})$, was described as a target of the immune response associated with IBD. Intriguingly, this protein is structurally related to a protein of P. gingivalis [40], against which increased autoantibody production was discovered in RA [41].

In addition to the clinical characteristics, some demographic and lifestyle factors of patients were taken into account. Enterobacteriales and Enterobacteriaceae (belonging to Gammaproteobacteria) were found to be associated with increasing age, while a number of taxa belonging to Betaproteobacteria (Alcaligenaceae, Burkholderiales, Sutterella, and S. wadsworthensis) were positively correlated with the habit of smoking.

When compared to previous reports, this study presents the advantage of having evaluated and compared, for the first time, the effects induced by synthetic and anti-TNF- $\alpha$ agents, in monotherapy and combination therapy, on gut dysbiosis in RA patients.

A limit to the current study is that the cross-sectional design can allow us to assess the presence of gut dysbiosis in RA patients who are treatment-naïve, under ETN, and/or MTX therapy; however, it does not give information on the predictive value of these changes in relation to RA clinical outcome and structural progression.

Overall, the current study revealed that RA is characterized by gut dysbiosis, some of which is associated to the inflammatory status of the disease, suggesting that the microbiota may play an important role in the promotion and clinical course of RA. Moreover, the partial restoration of a beneficial microbiota induced mainly by the anti-TNF- $\alpha$ ETN can contribute to the clinical efficacy of this agent. A deeper understanding of the alterations occurring in the gut microbiota of patients on different therapeutic regimens could help set up individualized and supportive therapeutic strategies providing patients with more effective and safe care.

\section{Materials and Methods}

\subsection{Study Population}

Forty-two RA patients, according to the European League Against Rheumatism (EULAR)/American College of Rheumatology (ACR) classification criteria [42] were recruited at the 
outpatient Division of Immunology and Rheumatology, S. Andrea Hospital, Sapienza University of Rome. Patients were divided into four groups according to current therapy: 11 patients were naïve to immunosuppressants, 11 patients were receiving MTX, 10 patients were receiving ETN, and 10 patients were receiving ETN plus MTX. Ten healthy subjects were used as controls (HCs).

The study was conducted according to the ethical guidelines of the 1975 Declaration of Helsinki. An informed consent was obtained by all the patients and the study was approved by the local ethical Committee (43/2013). All patients were receiving current therapy for at least three months and had to be naïve to other biotechnological drugs. Steroids and non-steroidal anti-inflammatory drugs (NSAIDs) had to be stopped at least seven days before the exams.

Any patients or HCs on antibiotics, consuming probiotics, or having a known history of inflammatory bowel disease or other autoimmune diseases were excluded.

DAS-28, ESR, CRP, RF, ACPA, and dietary and smoking habits were assessed the same day of stool sample collection.

Patients' clinical data are described in Table 1.

\subsection{Sample Collection and DNA Extraction}

Each participant collected a fresh stool sample in a collection tube filled with a DNA stabilization buffer (Canvax Biotech, Voden Medical Instruments, Meda, Italy). Then, $250 \mu \mathrm{L}$ of each sample was processed for microbial DNA extraction using the QIAamp DNA Stool Mini Kit (Qiagen, Milan, Italy) according to the manufacturer's protocol. DNA concentration and purity were assessed using a NanoDrop spectrophotometer (Thermo Scientific, Meda, Italy).

\subsection{Next-Generation Sequencing of Bacterial 16S Ribosomal RNA Gene}

The Illumina 16S Metagenomic Sequencing Library Preparation instructions were followed for high-throughput sequencing. Firstly, $12.5 \mathrm{ng}$ of each DNA extract was employed for the amplification of the V3-V4 hypervariable regions of the bacterial $16 \mathrm{~S}$ ribosomal RNA (rRNA) gene, using the following primers with Illumina adapters (underlined): forward primer: 5'-TCGTCGGCAGCGTCAGATGTGTATAAGAGACAGCCTACGGGNGGCWGCAG, reverse primer: 5'-GTCTCGTGGGCTCGGAGATGTGTATAAGAGACAGGACTACHVGGGTATCTAATCC, selected from Klindworth et al. [43]. The amplification reaction was carried out in the presence of the $2 \times$ KAPA HiFi HotStart Ready Mix (Roche, Milan, Italy) under the following conditions: initial denaturation at $95^{\circ} \mathrm{C}$ for $3 \mathrm{~min}$, followed by 25 cycles of denaturation at $95^{\circ} \mathrm{C}$ for $30 \mathrm{~s}$, primer annealing at $55^{\circ} \mathrm{C}$ for $30 \mathrm{~s}$, extension at $72{ }^{\circ} \mathrm{C}$ for $30 \mathrm{~s}$, with a final elongation at $72{ }^{\circ} \mathrm{C}$ for $5 \mathrm{~min}$. PCR amplicons were then purified by means of Agencourt AMPure XP beads (Beckman Coulter, Milan, Italy). The purified DNA products were then subjected to a further PCR to attach dual Illumina indices (Nextera XT Index Kit, Illumina Inc., San Diego, CA, USA) necessary for multiplexing. The reaction was performed under the following conditions: initial denaturation at $95^{\circ} \mathrm{C}$ for $3 \mathrm{~min}$, followed by eight cycles of denaturation at $95^{\circ} \mathrm{C}$ for $30 \mathrm{~s}$, primer annealing at $55^{\circ} \mathrm{C}$ for $30 \mathrm{~s}$, extension at $72{ }^{\circ} \mathrm{C}$ for $30 \mathrm{~s}$, with a final elongation at $72{ }^{\circ} \mathrm{C}$ for $5 \mathrm{~min}$. Following a further PCR purification, the eluted DNA products were quantified using the Qubit dsDNA BR Kit assay, diluted to a concentration of $4 \mathrm{nM}$, and pooled in equal proportion into a single library. Paired-end sequencing $(2 \times 300$ cycles $)$ was carried out on an Illumina MiSeq device (Illumina Inc.) according to the manufacturer's instructions. Sequences were demultiplexed based on index sequences, and FASTQ files were generated.

\subsection{Bioinformatic Analysis}

Sequence data were analyzed using the 16S Metagenomics App provided by BaseSpace software (version 1.0.1, Illumina Inc.) which performs taxonomic classification based on the Greengenes database (available online: http://greengenes.secondgenome.com/downloads/database/13_5). For each sample, the relative abundance of the top eight taxonomic classifications at each level (from phylum to 
species) was considered for statistical analysis. Moreover, the software calculated the Shannon index $(\alpha$-diversity) and the number of species (richness) found in each sample.

\subsection{Statistical Analysis}

Continuous variables are expressed as means \pm standard deviation (SD). Two-group comparisons were calculated using the Student's $t$-test. Correlation analyses were performed using Spearman rank correlation. Multiple logistic regression analyses with backward variable selection were applied to assess independent correlates of subject status (healthy/pathological). All statistical tests were two-tailed and $p<0.05$ was considered statistically significant. Statistical analyses were performed by using the R software, version 3.1.0 (10 April 2014)-B Spring Dance Copyright @ 2014 The R Foundation for Statistical Computing.

Supplementary Materials: Supplementary materials can be found at http:/ /www.mdpi.com/1422-0067/19/10/ 2938/s1.

Author Contributions: A.P.-D. and V.P. conceived the study; A.P.-D., C.P., V.P. and R.D. designed the experiments; C.P. performed the experiments; G.S., C.P., A.P.-D. and V.P. analyzed the data; A.P.-D., F.T. and V.P. contributed reagents/materials/analysis tools; S.S., M.L.S., B.L., M.S. and R.D.R. performed the medical examination of the RA patients; C.P., A.T., A.P.-D. and V.P. wrote the paper.

Funding: This research was supported by the "Ricerca Corrente RC1703GA31" and the "Ricerca Corrente RC1803GA30" granted by the Italian Ministry of Health to V.P.

Acknowledgments: We thank Riccardo Pracella for technical help.

Conflicts of Interest: The authors declare no conflict of interest.

\section{References}

1. Ursell, L.K.; Haiser, H.J.; Van Treuren, W.; Garg, N.; Reddivari, L.; Vanamala, J.; Dorrestein, P.C.; Turnbaugh, P.J.; Knight, R. The intestinal metabolome: An intersection between microbiota and host. Gastroenterology 2014, 146, 1470-1476. [CrossRef] [PubMed]

2. Maslowski, K.M.; Vieira, A.T.; Ng, A.; Kranich, J.; Sierro, F.; Yu, D.; Schilter, H.C.; Rolph, M.S.; Mackay, F.; Artis, D.; et al. Regulation of inflammatory responses by gut microbiota and chemoattractant receptor GPR43. Nature 2009, 461, 1282-1286. [CrossRef] [PubMed]

3. Rogier, R.; Koenders, M.I.; Abdollahi-Roodsaz, S. Toll-like receptor mediated modulation of T cell response by commensal intestinal microbiota as a trigger for autoimmune arthritis. J. Immunol. Res. 2015, 2015, 527696. [CrossRef] [PubMed]

4. Stecher, B.; Hardt, W.D. Mechanisms controlling pathogen colonization of the gut. Curr. Opin. Microbiol. 2011, 14, 82-91. [CrossRef] [PubMed]

5. Petersen, C.; Round, J.L. Defining dysbiosis and its influence on host immunity and disease. Cell Microbiol. 2014, 16, 1024-1033. [CrossRef] [PubMed]

6. Picchianti-Diamanti, A.; Rosado, M.M. D'Amelio, R. Infectious Agents and Inflammation: The Role of Microbiota in Autoimmune Arthritis. Front. Microbiol. 2018, 8, 2696. [CrossRef] [PubMed]

7. Asquith, M.; Elewaut, D.; Lin, P.; Rosenbaum, J.T. The role of the gut and microbes in the pathogenesis of spondyloarthritis. Best Pract. Res. Clin. Rheumatol. 2014, 28, 687-702. [CrossRef] [PubMed]

8. Ciccia, F.; Bombardieri, M.; Rizzo, A.; Principato, A.; Giardina, A.R.; Raiata, F.; Peralta, S.; Ferrante, A.; Drago, S.; Cottone, M.; et al. Over-expression of paneth cell-derived anti-microbial peptides in the gut of patients with ankylosing spondylitis and subclinical intestinal inflammation. Rheumatology 2010, 49, 2076-2083. [CrossRef] [PubMed]

9. Picchianti Diamanti, A.; Lagana, B.; Cox, M.C.; Pilozzi, E.; Amodeo, R.; Bove, M.; Markovic, M.; di Rosa, R.; Salemi, S.; Sorgi, M.L.; et al. TCD4 pos lymphocytosis in rheumatoid and psoriatic arthritis patients following TNF $\alpha$ blocking agents. J. Transl. Med. 2017, 15, 38. [CrossRef] [PubMed]

10. Ciccia, F.; Ferrante, A.; Guggino, G.; Triolo, G. The role of the gastrointestinal tract in the pathogenesis of rheumatic diseases. Best Pract. Res. Clin. Rheumatol. 2016, 30, 889-900. [CrossRef] [PubMed]

11. Panebianco, C.; Andriulli, A.; Pazienza, V. Pharmacomicrobiomics: Exploiting the drug-microbiota interactions in anticancer therapies. Microbiome 2018, 6, 92. [CrossRef] [PubMed] 
12. Zhang, X.; Zhang, D.; Jia, H.; Feng, Q.; Wang, D.; Liang, D.; Wu, X.; Li, J.; Tang, L.; Li, Y.; et al. The oral and gut microbiomes are perturbed in rheumatoid arthritis and partly normalized after treatment. Nat. Med. 2015, 21, 895-905. [CrossRef] [PubMed]

13. Enright, E.F.; Gahan, C.G.; Joyce, S.A.; Griffin, B.T. The impact of the gut microbiota on drug metabolism and clinical outcome. Yale J. Biol. Med. 2016, 89, 37.

14. Quirke, A.M.; Lugli, E.B.; Wegner, N.; Hamilton, B.C.; Charles, P.; Chowdhury, M.; Ytterberg, A.J.; Zubarev, R.A.; Potempa, J.; Culshaw, S.; et al. Heightened immune response to autocitrullinated Porphyromonas gingivalis peptidylarginine deiminase: A potential mechanism for breaching immunologic tolerance in rheumatoid arthritis. Ann. Rheum. Dis. 2014, 73, 263-269. [CrossRef] [PubMed]

15. Wegner, N.; Wait, R.; Sroka, A.; Eick, S.; Nguyen, K.A.; Lundberg, K.; Kinloch, A.; Culshaw, S.; Potempa, J.; Venables, P.J. Peptidylarginine deiminase from Porphyromonas gingivalis citrullinates human fibrinogen and alpha-enolase: Implications for autoimmunity in rheumatoid arthritis. Arthritis Rheum. 2010, 62, 2662-2672. [CrossRef] [PubMed]

16. Smolen, J.S.; Landewe, R.; Bijlsma, J.; Burmester, G.; Chatzidionysiou, K.; Dougados, M.; Nam, J.; Ramiro, S.; Voshaar, M.; van Vollenhoven, R.; et al. EULAR recommendations for the management of rheumatoid arthritis with synthetic and biological disease-modifying antirheumatic drugs: 2016 Update. Ann. Rheum. Dis. 2017, 76, 960-977. [CrossRef] [PubMed]

17. Bhat, M.; Pasini, E.; Copeland, J.; Angeli, M.; Husain, S.; Kumar, D.; Renner, E.; Teterina, A.; Allard, J.; Guttman, D.S.; et al. Impact of immunosuppression on the metagenomic composition of the intestinal microbiome: A systems biology approach to post-transplant diabetes. Sci. Rep. 2017, 7, 10277. [CrossRef] [PubMed]

18. Liu, X.; Zou, Q.; Zeng, B.; Fang, Y.; Wei, H. Analysis of fecal Lactobacillus community structure in patients with early rheumatoid arthritis. Curr. Microbiol. 2013, 67, 170-176. [CrossRef] [PubMed]

19. Liu, X.; Zeng, B.; Zhang, J.; Li, W.; Mou, F.; Wang, H.; Zou, Q.; Zhong, B.; Wu, L.; Wei, H.; et al. Role of the gut microbiome in modulating arthritis progression in mice. Sci Rep. 2016, 6, 30594. [CrossRef] [PubMed]

20. Alipour, B.; Homayouni-Rad, A.; Vaghef-Mehrabany, E.; Sharif, S.K.; Vaghef-Mehrabany, L.; Asghari-Jafarabadi, M.; Nakhjavani, M.R.; Mohtadi-Nia, J. Effects of Lactobacillus casei supplementation on disease activity and inflammatory cytokines in rheumatoid arthritis patients: A randomized double-blind clinical trial. Int. J. Rheum. Dis. 2014, 17, 519-527. [PubMed]

21. Amdekar, S.; Singh, V.; Singh, R.; Sharma, P.; Keshav, P.; Kumar, A. Lactobacillus casei reduces the inflammatory joint damage associated with collagen-induced arthritis (CIA) by reducing the pro-inflammatory cytokines: Lactobacillus casei: COX-2 inhibitor. J. Clin. Immunol. 2011, 31, 147-154. [CrossRef] [PubMed]

22. Kano, H.; Kaneko, T.; Kaminogawa, S. Oral intake of Lactobacillus delbrueckii subsp. bulgaricus OLL1073R-1 prevents collagen-induced arthritis in mice. J. Food Prot. 2002, 65, 153-160. [CrossRef] [PubMed]

23. So, J.S.; Kwon, H.K.; Lee, C.G.; Yi, H.J.; Park, J.A.; Lim, S.Y.; Hwang, K.C.; Jeon, Y.H.; Im, S.H. Lactobacillus casei suppresses experimental arthritis by down-regulating T helper 1 effector functions. Mol. Immunol. 2008, 45, 2690-2699. [CrossRef] [PubMed]

24. Hatakka, K.; Martio, J.; Korpela, M.; Herranen, M.; Poussa, T.; Laasanen, T.; Saxelin, M.; Vapaatalo, H.; Moilanen, E.; Korpela, R. Effects of probiotic therapy on the activity and activation of mild rheumatoid arthritis-A pilot study. Scand. J. Rheumatol. 2003, 32, 211-215. [CrossRef] [PubMed]

25. Pineda Mde, L.; Thompson, S.F.; Summers, K.; de Leon, F.; Pope, J.; Reid, G. A randomized, double-blinded, placebo-controlled pilot study of probiotics in active rheumatoid arthritis. Med. Sci. Monit. 2011, 17, CR347-CR354. [PubMed]

26. Chen, J.; Wright, K.; Davis, J.M.; Jeraldo, P.; Marietta, E.V.; Murray, J.; Nelson, H.; Matteson, E.L.; Taneja, V. An expansion of rare lineage intestinal microbes characterizes rheumatoid arthritis. Genome Med. 2015, 8, 43. [CrossRef] [PubMed]

27. Wu, X.; Liu, J.; Xiao, L.; Lu, A.; Zhang, G. Alterations of gut microbiome in rheumatoid arthritis. Osteoarthr. Cartil. 2017, 25, S287-S288. [CrossRef]

28. Arvonen, M.; Berntson, L.; Pokka, T.; Karttunen, T.J.; Vahasalo, P.; Stoll, M.L. Gut microbiota-host interactions and juvenile idiopathic arthritis. Pediatr. Rheumatol. Online J. 2016, 14, 44. [CrossRef] [PubMed]

29. Cao, Y.; Shen, J.; Ran, Z.H. Association between Faecalibacterium prausnitzii reduction and inflammatory bowel disease: A meta-analysis and systematic review of the literature. Gastroenterol. Res. Pract. 2014, 2014, 872725. [CrossRef] [PubMed] 
30. Sokol, H.; Pigneur, B.; Watterlot, L.; Lakhdari, O.; Bermudez-Humaran, L.G.; Gratadoux, J.J.; Blugeon, S.; Bridonneau, C.; Furet, J.P.; Corthier, G.; et al. Faecalibacterium prausnitzii is an anti-inflammatory commensal bacterium identified by gut microbiota analysis of Crohn disease patients. Proc. Natl. Acad. Sci. USA 2008, 105, 16731-16736. [CrossRef] [PubMed]

31. Lopez, P.; de Paz, B.; Rodriguez-Carrio, J.; Hevia, A.; Sanchez, B.; Margolles, A.; Suarez, A. Th17 responses and natural IgM antibodies are related to gut microbiota composition in systemic lupus erythematosus patients. Sci. Rep. 2016, 6, 24072. [CrossRef] [PubMed]

32. Vijayakumar, S.; Menakha, M. Pharmaceutical applications of cyanobacteria-A review. J. Acute Med. 2015, 5, 15-23. [CrossRef]

33. Roediger, W.E.; Moore, J.; Babidge, W. Colonic sulfide in pathogenesis and treatment of ulcerative colitis. Dig. Dis. Sci. 1997, 42, 1571-1579. [CrossRef] [PubMed]

34. Rizzatti, G.; Lopetuso, L.R.; Gibiino, G.; Binda, C.; Gasbarrini, A. Proteobacteria: A common factor in human diseases. BioMed Res. Int. 2017, 2017, 9351507. [CrossRef] [PubMed]

35. Muniz-Pedrogo, D.A.; Chen, J.; Hillmann, B.M.; Jeraldo, P.; Saffouri, G.; Al-Ghalith, G.A.; Friton, J.; Taneja, V.; Davis, J.M.; Knights, D.; et al. Gut microbial markers of arthritis including inflammatory bowel disease associated arthropathy. Gastroenterology 2018, 154, S-586. [CrossRef]

36. Ramos-Romero, S.; Hereu, M.; Atienza, L.; Casas, J.; Jauregui, O.; Amezqueta, S.; Dasilva, G.; Medina, I.; Nogues, M.R.; Romeu, M.; et al. Mechanistically different effects of fat and sugar on insulin resistance, hypertension, and gut microbiota in rats. Am. J. Physiol. Endocrinol. Metab. 2018, 314, E552-E563. [CrossRef] [PubMed]

37. Pedersen, C.; Ijaz, U.Z.; Gallagher, E.; Horton, F.; Ellis, R.J.; Jaiyeola, E.; Duparc, T.; Russell-Jones, D.; Hinton, P.; Cani, P.D.; et al. Fecal Enterobacteriales enrichment is associated with increased in vivo intestinal permeability in humans. Physiol. Rep. 2018, 6, e13649. [CrossRef] [PubMed]

38. Bjarnason, I.; Williams, P.; So, A.; Zanelli, G.D.; Levi, A.J.; Gumpel, J.M.; Peters, T.J.; Ansell, B. Intestinal permeability and inflammation in rheumatoid arthritis: Effects of non-steroidal anti-inflammatory drugs. Lancet 1984, 2, 1171-1174. [CrossRef]

39. Jangi, S.; Gandhi, R.; Cox, L.M.; Li, N.; von Glehn, F.; Yan, R.; Patel, B.; Mazzola, M.A.; Liu, S.; Glanz, B.L.; et al. Alterations of the human gut microbiome in multiple sclerosis. Nat. Commun. 2016, 7, 12015. [CrossRef] [PubMed]

40. Wei, B.; Dalwadi, H.; Gordon, L.K.; Landers, C.; Bruckner, D.; Targan, S.R.; Braun, J. Molecular cloning of a Bacteroides caccae TonB-linked outer membrane protein identified by an inflammatory bowel disease marker antibody. Infect. Immun. 2001, 69, 6044-6054. [CrossRef] [PubMed]

41. Johansson, L.; Sherina, N.; Kharlamova, N.; Potempa, B.; Larsson, B.; Israelsson, L.; Potempa, J.; Rantapaa-Dahlqvist, S.; Lundberg, K. Concentration of antibodies against Porphyromonas gingivalis is increased before the onset of symptoms of rheumatoid arthritis. Arthritis Res. Ther. 2016, 18, 201. [CrossRef] [PubMed]

42. Aletaha, D.; Neogi, T.; Silman, A.J.; Funovits, J.; Felson, D.T.; Bingham, C.O., III; Birnbaum, N.S.; Burmester, G.R.; Bykerk, V.P.; Cohen, M.D.; et al. 2010 rheumatoid arthritis classification criteria: An american college of rheumatology/European league against rheumatism collaborative initiative. Ann. Rheum. Dis. 2010, 69, 1580-1588. [CrossRef] [PubMed]

43. Klindworth, A.; Pruesse, E.; Schweer, T.; Peplies, J.; Quast, C.; Horn, M.; Glockner, F.O. Evaluation of general $16 \mathrm{~S}$ ribosomal RNA gene PCR primers for classical and next-generation sequencing-based diversity studies. Nucleic Acids Res. 2013, 41, e1. [CrossRef] [PubMed]

(C) 2018 by the authors. Licensee MDPI, Basel, Switzerland. This article is an open access article distributed under the terms and conditions of the Creative Commons Attribution (CC BY) license (http:/ / creativecommons.org/licenses/by/4.0/). 